\title{
Observational Properties of Triple Galaxies
}

\author{
I. D. Karachentsev
}

Special Astrophysical Observatory, Russia

\begin{abstract}
.
This report presents basic observational parameters for galaxy triplets selected by criterion of their isolation on the sky (=Catalog of Triple Galaxies, Karachentseva et al, 1979). The CTG sample is compared with a sample of wide triple systems from Nearby Galaxies Catalog (Tully, 1988) as well from a new whole-sky catalog of small galaxy groups in the Local Supercluster (Makarov \& Karachentsev, 2000). For all the samples their medians of the virial mass-to-luminosity ratio lie in a narrow range, $31<M_{\text {vir }} / L<36\left(M_{\odot} / L_{\odot}\right)$, while the medians of the crossing time occupy a wider range, $0.07<T_{\text {cross }}<0.37$ (in $1 / H$ units).
\end{abstract}

\section{Introduction}

Groups of galaxies occupy an intermediate position in the spectrum of galaxy populations between individual and binary galaxies on the one hand, and rich clusters on the other hand. According to de Vaucouleurs (1975), groups contain nearly half of the galaxies in the universe. Studies of groups bear upon a number of crucial questions in astronomy and cosmology. However, the impact of these studies is moderated by several statistical problems in the identification and analysis of systems with small numbers of members. As is well known, optical and $\mathrm{HI}$ mass determinations of galaxy rotational curves suggest that dark matter may reside in large halos around individual giant galaxies, typically extending to 100-200 kpc. Since the first observations by Zwicky in the 1930's, much evidence has also been accumulated for the existence of a large amount of dark matter in rich clusters of galaxies with sizes between 1 and $3 \mathrm{Mpc}$. It is indicated by the high velocity dispersion of member galaxies and the high temperature of the intragalactic gas in clusters. Contrary to individual galaxies and clusters of galaxies, the mass estimation for groups of galaxies presents a thorny problem. This is mainly because of poor observational material that can be used for this purpose. In this report I discuss observational properties of triple systems of galaxies as a special type of group with the smallest number of members. Triplets reveal all the complexity of the group statistics, dynamics and evolution.

\section{Catalogue of Triple Galaxies}

The Catalogue of Triple Galaxies (hereafter CTG) was the first published list of isolated galaxy triplets (Karachentseva et al., 1979). It contained 83 triple 

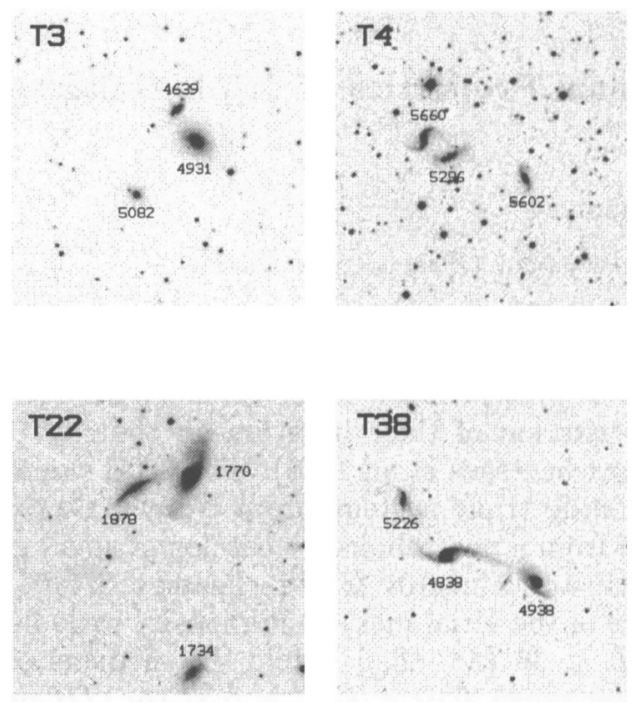

Figure 1. The four panels show images of the triplets: nos. 3, 4, 22, 38 from the digitized POSS-I. Each panel is 6 arcmin on a side. North is at the top, and east is to the left. Radial velocities of the triple components (in $\mathrm{km} \mathrm{s}^{-1}$ are indicated with numbers.

systems with galaxies of visual magnitude $m_{p} \leq 15.7$ and declinations $\delta>-3^{\circ}$. The galaxies were selected on the basis of the Palomar Sky Atlas and the Catalogue of Zwicky et al. (1968). Three galaxies were considered to form an isolated system if their 'significant' neighbors were at least three times as far away from them as the components of the triplet were from one another. 'Significant neighbors' were neighboring galaxies whose angular diameters differed by a factor no larger than two times the diameter of a triplet member. This empirically chosen isolation criterion provided the surface density contrast of the triplet over a local background to be $>10$. Fig.1 gives examples of some triplets.

Triple-system membership of a galaxy was determined without regard to its radial velocity. Therefore a system can be considered isolated only in projection on the sky and, generally, not in three-dimensional space. The radial velocities of all galaxies of CTG have been measured mainly with the 6-m telescope of Special Astrophysical Observatory. Karachentseva et al., (1988) give the following data. for CTG systems:

1. Number of the triplet and letter designation of its components;

2. Satisfaction of the isolation criterion (Is) for each component with + as yes and - as no; 
3,4. Right ascension and declination at epoch 1950.0;

5,6 . Galactic coordinates $l^{I I}, b^{I I}$;

7. Angular diameter $a_{25}$ (major axis) in arc minutes corresponding to $25 \mathrm{~m}$ per square arc second surface brightness;

8. Minor-to-major axis ratio $b_{25} / a_{25}$ of projected ellipse;

9. Visual magnitude of components $m_{\mathrm{Ho}}^{c}$ reduced to Holmberg's system with the corrections for the galactic extinction, K-correction and internal absorption;

10. Apparent angular separations $x_{i, k}$ of the components in arcmin;

11. Configuration (C) of the triplet: $\mathrm{D}$ - hierarchical (one of the sides of the apparent triangle is $1 / 3$ or less of each of the two other sides); $\mathrm{L}$ - linear (one of the angles of the apparent triangle is more than $150^{\circ}$ ); $\mathrm{T}$ - triangle;

12. Morphological type (Ty) of the member galaxy, one of 6 classes: $\mathrm{E}, \mathrm{S} 0$, $\mathrm{Sa}, \mathrm{Sb}, \mathrm{Sc}, \mathrm{Sm}$;

13. Spectral index (Sp) of the member galaxy from: A - pure absorption, to $\mathrm{S}$ - strong emissions;

14. Radial velocity $\left(v_{o}\right)$ in $\mathrm{km} \mathrm{s}^{-1}$ corrected for the motion of the Sun and internal error $\left(\sigma_{v}\right)$ of the radial velocity measurements;

\section{Dynamical parameters of the triplets}

Each triplet as a dynamical system is characterized by the following parameters: 1. The rms velocity of the member galaxies relative to the center,

$$
s_{v}^{2}=(1 / 3) \sum\left(v_{k}-<v>\right)^{2}, k=1,2,3 ;
$$

2. The mean harmonic separation in projection,

$$
r_{H}=\left[(1 / 3) \sum r_{i k}^{-1}\right]^{-1}
$$

where the projection of the separation of the two galaxies in a triplet is

$$
r_{i k}=x_{i k}<v>H^{-1}
$$

$x_{i k}$ are the projected angular separations, $H=75 \mathrm{~km} \mathrm{~s}^{-1} \mathrm{Mpc}^{-1}$, is the Hubble constant.

3. The luminosity of a galaxy in solar units,

$$
L_{k}=10^{0.4\left(5.40-M_{k}\right)}
$$

where the absolute magnitude $M_{k}$ is determined from the mean velocity $\langle v\rangle$ and the apparent magnitude corrected for the galactic extinction and internal absorption in given galaxy.

4. The dimensionless crossing time of the triplets expressed in terms of Hubble time $\left(H^{-1}\right)$ is

$$
\tau=2 H\left(r_{H} / s_{v}\right)
$$


5. The virial mass-to-light ratio for a triple system,

$$
f=M_{v i r}\left[\sum L_{i}\right]^{-1}, i=1,2,3
$$

in solar units. Here

$$
M_{v i r}=3 \pi N(N-1)^{-1} G^{-1} s_{v}^{2} r_{H}, \quad N=3,
$$

is the 'virial' mass of a triplet where $G$ is the gravitational constant.

6. The statistically unbiased estimate of the same ratio,

$$
f^{c}=f\left[1-\left(2 \sigma_{v}^{2}\right) /\left(3 s_{v}^{2}\right)\right]
$$

where $\sigma_{v}$ is the rms error of the radial velocity measurements.

\section{Velocity and separation distributions}
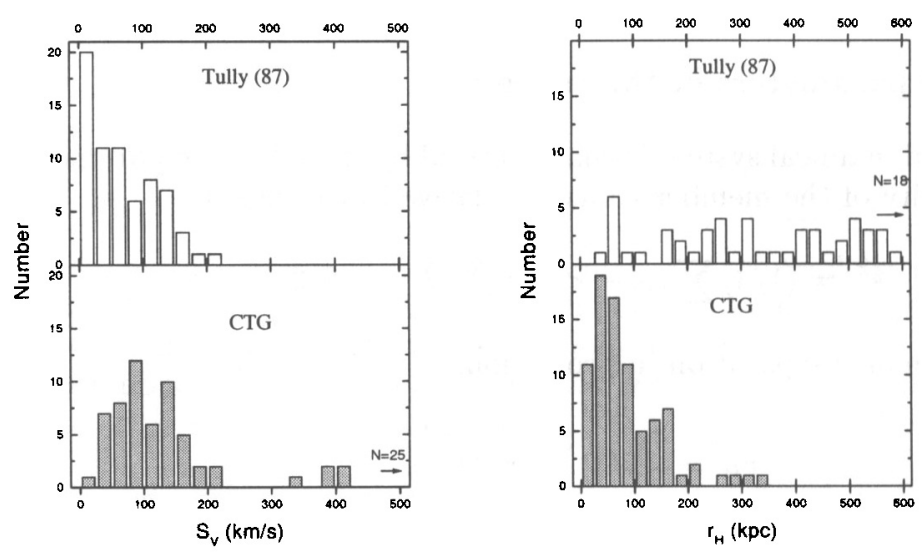

Figure 2: The distribution of the radial velocity dispersion for the CTG (shaded) and Tully's triple systems. Among 83 CTG triplets 25 have $s_{v}>500 \mathrm{~km} \mathrm{~s}^{-1}$.

Figure 3: Number distribution of triple system from CTG (shaded) and Tully's sample versus the mean harmonic projected separation. Eighteen Tully's triple systems have $r_{H}>600 \mathrm{kpc}$.

Fig. 2 (bottom) represents the distribution of the rms velocity $\left(s_{v}\right)$ of the CTG systems. It is seen that the distribution is asymmetric with the main maximum at about $100 \mathrm{~km} \mathrm{~s}^{-1}$, and a long tail extending to $10000 \mathrm{~km} \mathrm{~s}^{-1}$. The upper part of Fig.2 shows a similar distribution for 69 triple systems which are taken from the Nearby Galaxies Catalog by Tully $(1987,1988)$ (see below). It is well-known that in statistical distributions with large asymmetry, the sample mean values are sensitive to a few highest members in magnitude in the tail. 
In such cases, the sample median value is more stable statistically. For the velocity distributions of the CTG, the median is $133 \mathrm{~km} \mathrm{~s}^{-1}$, while for Tully's triple systems $58 \mathrm{~km} \mathrm{~s}^{-1}$ only.

Fig. 3 shows the mean harmonic projected separation distribution. For the CTG triplets (lower) it has a median of $63 \mathrm{kpc}$, while for Tully's triple systems the median is $430 \mathrm{kpc}$.

The distribution of the triplets in the $s_{v}$ versus $r_{H}$ diagram is presented in Fig. 4. On the whole, a trend is seen for the velocity dispersion to decrease from close to wide systems.

\section{The problems of selection and contamination}
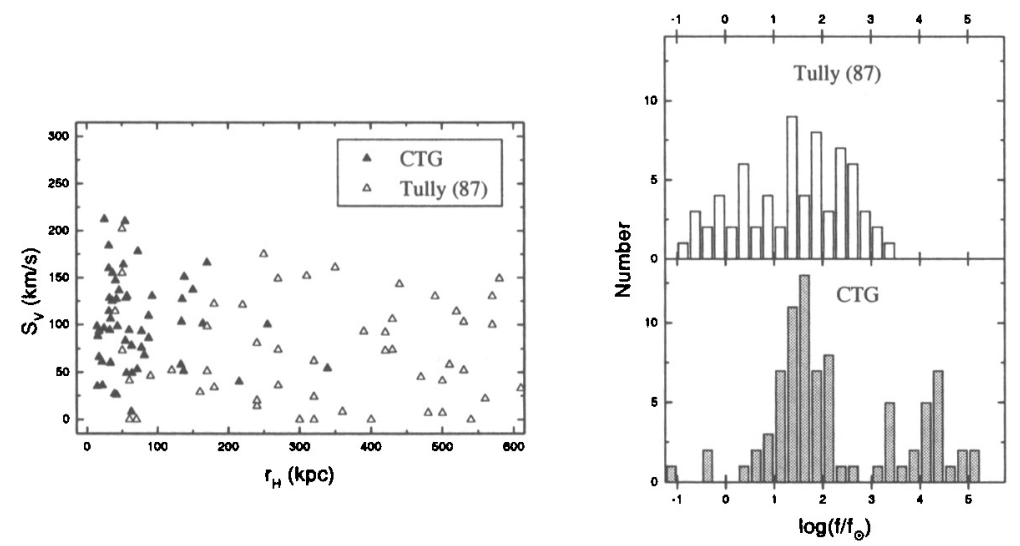

Figure 4 (left): The distribution of CTG and Tully's triplets in $s_{v}$ versus $r_{H}$ diagram.

Figure 5 (right): Distribution histograms over mass-to-light ratio of triple systems from CTG and Tully's samples.

There are indications that the CTG sample contains a considerable fraction of nonisolated and false triple systems. To investigate this problem, a computer simulation was carried out by Karachentsev \& Shcherbanovsky (1978). Based on more or less likely suggestions concerning the luminosity function of galaxies, multiplicity function for galaxy systems, and distribution of galaxies within a system according to coordinates and velocities, they generated a large number of artifical "Palomar Sky Atlas charts". The same procedure of selection of triple systems was then applied to these simulated charts. As a result, 71 apparently isolated triple systems were found among 10000 simulated galaxies brighter than 15.7. The main conclusions from the analysis of the pseudo-CTG sample may be summarized as follows:

1. The isolation criterion proves to be strongly selective favoring the closest triple systems as well the most luminous ones. 
2. The fraction of triple galaxies in a space, assumed to be $15 \%$, leads to a catalogic fraction about $2 \%$ only.

3. Among the simulated triple systems satisfying the isolation criterion the following are presented: $7 \%$ are true (physical) triplets, $7 \%$ are "optical" systems where galaxies are completely unrelated to each other and the visible appearance of them as triplets is due to projection effects, $42 \%$ are "semi-optical" systems which are the result of projection of a background/foreground galaxy on the region near a binary galaxy, and $44 \%$ are members of one and the same group or cluster satisfying the isolation condition in projection only.

4. Although the distributions of the rms velocity for the optical and physical systems overlap slightly, there is no great difficulty in distinguishing between them. The simulated velocity distribution reveals that the optical triplets dominate in the range $s_{v}>300 \mathrm{~km} \mathrm{~s}^{-1}$. The situation is different with the triplets whose member galaxies belong to groups. They overlap with the true isolated triplets to such a great extent that there is no simple way of distinguishing them, if one uses only the velocity distributions.

\section{Virial mass-to-light distributions}

We define here by mass the combination of observational characteristics of the CTG systems with the dimension of mass described above. Fig. 5 shows the 'virial' mass-to-light distribution on a logarithmic scale of quantity $f$. Three feature are seen in the histogram:

1. the wide range of the mass-to-light ratio which covers 7 decades;

2 . the position of the main maximum near $\approx 30$, in solar units;

3 . the presence of the secondary rise in the range $10^{3}-10^{5}$.

According to the results of computer simulations, the range of the secondary maximum is occupied by optical triplets.

\section{Table 1. Median values of virial mass to luminosity ratio of} 83 triplets

\begin{tabular}{lcccccc}
\hline \hline Method of & \multicolumn{2}{c}{ Entire Sample } & \multicolumn{2}{c}{ With $f<10^{3}$} & \multicolumn{2}{c}{ With $s_{v}<300 \mathrm{~km} \mathrm{~s}^{-1}$} \\
evaluation & $f_{\text {med }}$ & $f_{\text {med }}^{c}$ & $f_{\text {med }}$ & $f_{\text {med }}^{c}$ & $f_{\text {med }}$ & $f_{\text {med }}^{c}$ \\
\hline Virial & 68 & 55 & 34 & 29 & 31 & 27 \\
Weighted Virial & 50 & 46 & 31 & 29 & 31 & 27 \\
Pairwised & 54 & 49 & 28 & 24 & 22 & 20 \\
\hline Isolated Pairs & 8 & 7 & 7 & 5 & 6 & 4 \\
\hline
\end{tabular}

The figures for the median mass-to-light ratios are presented in Table 1. Along with the entire sample, two sub-samples are analyzed in a similar way to try to exclude optical systems. One of them assumes that 25 triplets with mass-to-light ratios $>10^{3}$, in solar units, are optical. Another assumes that 30 triplets with the rms velocity $s_{v}>300 \mathrm{~km} \mathrm{~s}^{-1}$ are optical. The last line in the table gives, for comparison, the medians of the corresponding distributions for the isolated binary galaxies (Karachentsev 1987). The data of Table 1 suggest the following inferences: 
1. Accounting for or neglecting the errors in radial velocity measurements does not affect essentially the median values of the mass-to-light ratio;

2. The typical figures for the median mass-to-light ratios are within a rather narrow range, $20-30$, in solar units;

3. The medians are insensitive to the procedure of definition used to estimate the mass-to-light ratio;

4. The triplets reveal a definite excess of mass-to-light ratio over isolated binary galaxies; for the entire sample, as well as for the sub-samples, this ratio is about 5 times larger for the triplets than for the binaries.

A special analysis (Karachentsev et al. 1989) demonstrates that the median of the mass-to-light ratio is stable also against configurations of the triplets: it is practically the same for sub-samples arranged in accordance with the type of configurations (see above); in particular, the hierarchical configurations (dynamically most long-lived) do not differ in this sense from other configurations. It is also insensitive to the degree of isolation of the triplets, as well as the distance to the system. There is practically no correlation between the mass-to-light ratio and the dominant morphological type of member galaxies in the triplets. As it was found above, a typical mass-to-light ratio for the CTG systems is 20 to 30 , in solar units, and a typical size of the systems is about $70 \mathrm{kpc}$. But is there any dependence of mass-to-light ratios on sizes for individual CTG systems? This question is essential for studies of the dark matter problem.

Fig. 6 shows the $f$-versus- $r_{H}$ diagram for the triplets. Note that most of the CTG triplets are concentrated in the region of $r_{H}<100 \mathrm{kpc}, f<70$, while Tully's triple systems extend much farther. A possible increase in $f$ with size that would be expected in some models of dark matter is very small and of low statistical significance.
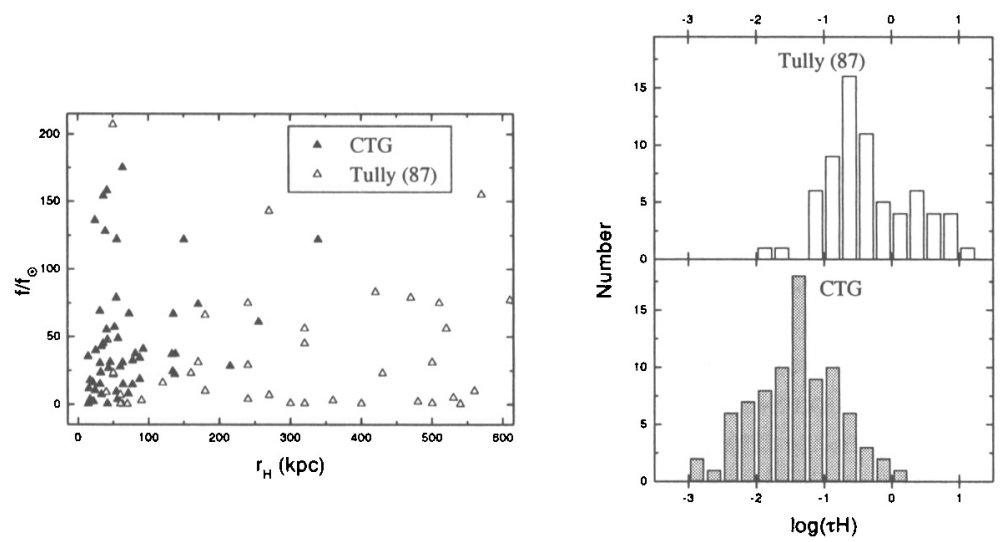

Figure 6 (left): Mass-to-luminosity ratio versus the mean harmonic projected radius.

Figure 7 (right): Distribution with respect to $\log$ crossing time in the Hubble time units. 


\section{Crossing-time distribution}

The system's crossing time $\tau$ was defined above with the use of the mean harmonic radius of the triplet and its rms velocity. The $\log \tau$ distribution is shown in the lower part of Fig. 7, where the Hubble time is $\log \tau=0$. It looks fairly symmetric, and its median is -1.40 . These data indicate that the typical crossing time for the CTG triplets is an order of magnitude less that the Hubble time. If the systems are bound, they also show that $90 \%$ of the triplets have $\tau<0.5$, which means that their evolution should be at an advanced dynamical stage when their ensemble reaches a 'virialized' statistical state. However, as one can find from the upper part of Fig. 7, a half of Tully's triple systems seem to be dynamically young unvirilized systems.

\section{Wide triplets}

As it has already been noted above, the isolation criterion used in the CTG has a strong selectivity, especially, for the wide low luminosity systems. Hence, we may expect that there exist a lot of wide galaxy triplets, which do not satisfy the CTG criterion, with a typical dimension $>100 \mathrm{kpc}$. To have an idea of their properties, we selected triple systems from some known lists of groups of galaxies. We used the galaxy group lists issued by Geller \& Huchra (1983), Vennik (1986), Tully (1987), Magtessian (1988), as well a new catalog of small groups in the Local Supercluster (Makarov \& Karachentsev, 2000). These authors applied various procedures of group sampling. A detailed comparison of various selection criteria was presented by Nolthenius (1993), Garcia et al.(1993), Karachentsev (1994), Bahcall et al. (1995).

The basic parameters of triple galaxy systems from these lists are given in Table 2. Its columns contain:

(1) the sample considered,

(2) the total number of triple systems,

(3) the median of the rms velocity,

(4) the median of the harmonic radius,

(5) the median of the total triplet luminosity,

(6) the median of virial mass-to-luminosity ratio,

(7) the median of crossing time in the cosmologic time units,

(8) a fraction of galaxies in the sample which belong to triple systems,

(9) some parameters of the sampling.

All the data are reduced to the Hubble constant $H=75 \mathrm{~km} \mathrm{~s}^{-1} \mathrm{Mpc}^{-1}$.

The data of Table 2 are consistent with the idea that besides compact isolated triplets there are a lot of wider triple systems with a typical harmonic radius in the range $160-430 \mathrm{kpc}$, whose relative number per unit volume exceeds the number of CTG triplets by 5-8 times. The wider triple galaxy systems have a typical rms velocity of $40-100 \mathrm{~km} \mathrm{~s}^{-1}$ and the median virial- mass-to-luminosity ratio about 20-40 in solar units. (Here the sample of Geller \& Huchra (1983) was disregarded because of its strong contamination with false systems). Due to the long median crossing time, $0.15-0.37$, a significant part of the wide triple systems should be considered as young unvirilized ones. 
Table 2. Basic median values for triple galaxies from different samples

\begin{tabular}{|c|c|c|c|c|c|c|c|c|}
\hline Sample & $\mathrm{N}_{3}$ & $\begin{array}{c}s_{v} \\
\mathrm{kms}^{-1}\end{array}$ & $\begin{array}{l}r_{H} \\
\mathrm{kpc} \\
\end{array}$ & $\begin{array}{c} \\
1010 \\
\end{array}$ & $\begin{array}{c}M_{v} / \bar{L} \\
M_{\odot} / L_{\odot} \\
\end{array}$ & $\tau \overline{\tau H}$ & $\overline{Q_{3}}$ & Selection parameters \\
\hline 1 & 2 & 3 & 4 & 5 & 6 & 7 & 8 & 9 \\
\hline $\begin{array}{l}\text { Geller \& Huchra, } \\
1983\end{array}$ & 58 & 112 & $6 \overline{15}$ & - & 276 & 1.05 & $7 \%$ & $\begin{array}{l}\Delta V<400 \mathrm{kms}^{-\mathrm{T}} \\
\Delta R<0.52 \mathrm{Mpc} \\
N_{t}=2390\end{array}$ \\
\hline Vennik, 1986 & 23 & 56 & 160 & 1.7: & 73 & 0.19 & $4 \%$ & $\begin{array}{l}\Delta V<300 \mathrm{kms}^{-1} \\
\lg \rho>\rho_{0} \\
N_{t}=1700\end{array}$ \\
\hline Tully, 1987 & 69 & 58 & 430 & 4.8 & 36 & 0.37 & $9 \%$ & $\begin{array}{l}\Delta V<300 \mathrm{~km} \mathrm{~s}^{-1} \\
\lg \rho>9.4 \\
N_{t}=2367\end{array}$ \\
\hline Magtessian, 1988 & 53 & 100 & 180 & - & 20 & 0.16 & $7 \%$ & $\begin{array}{l}\Delta V<400 \mathrm{~km} \mathrm{~s}^{-1} \\
E>E_{0} \\
N_{t}=2400\end{array}$ \\
\hline $\begin{array}{l}\text { Karachentseva et al, } \\
1988 \text { (=CTG) }\end{array}$ & 83 & 133 & 63 & 7.4 & 67 & 0.04 & $1 \%$ & $\begin{array}{l}x_{i 1}>3 x_{12} \\
N_{t}=27840\end{array}$ \\
\hline $\begin{array}{l}\text { CTG, but with } \\
s_{v}<300 \mathrm{~km} \mathrm{~s}^{-1}\end{array}$ & 53 & 100 & 55 & 7.6 & 31 & 0.07 & $1 \%$ & \\
\hline $\begin{array}{l}\text { Makarov \& } \\
\text { Karachentsev, } 2000\end{array}$ & 156 & 41 & 169 & 2.3 & 32 & 0.15 & $8 \%$ & $\begin{array}{l}\tau<1, E<0, \\
V_{0}<3000 \\
N_{t}=5976 \kappa=3\end{array}$ \\
\hline
\end{tabular}

We believe that the most realistic parameters of wide galaxy triplets come from a new catalog of small groups in the Local Supercluster (Makarov \& Karachentsev, 2000), because its sampling criterion is apparently more efficient than the others. Unlike the "friends-of- friends" criterion (Geller \& Huchra, 1983 ) and the "dendrogram" approach (Tully, 1987), the selection algorithm, used in the new catalog, takes into account individual properties of clustering galaxies, which avoids a majority of spurious "groups" procuced by simplified selection criteria.

\section{Conclusions}

We have considered a sample of 83 isolated triplets of galaxies with a local overdensity on the sky of more than an order of magnitude. After removing false optical systems, the sample has the following median parameters:

projected harmonic radius : $55 \mathrm{kpc}$,

rms velocity : $100 \mathrm{kms}^{-1}$,

total luminosity : $7.6 \times L_{\odot}$,

and dimensionless crossing time : 0.07 .

Using various ways to estimate virial mass-to-luminosity ratio, we found it to range from 20 to $30 f_{\odot}$. 
The sample of isolated (=CTG) triplets was compared with two samples of wide triple systems from Tully (1987) catalog $(\mathrm{N}=69)$ and a new catalog of small groups in the Local Supercluster $(N=156)$. Even though the wide triple systems are 3 -- 7 times more extended than the CTG triplets and they have a median crossing time of $\sim 0.25$, their median virial mass-to-luminosity ratio, 36 and $32 f_{\odot}$ respectively in the two samples, agree well with the CTG value. This value is one order of magnitude less than for the Geller \& Huchra (1983) triple systems, but is $4-5$ times as large as the median $M / L$ ratio for the isolated galaxy pairs or for individual galaxies.

Apparently, the $M_{v} / L$ ratio remains roughly constant with a radius out to a few hundred kiloparsecs, which limits the size of a possible dark halo in triple galaxies.

The relative number of compact triplets in galaxy catalogs $Q_{3} \sim 1 \%$ increases to $7-9 \%$ when a population of wide triplets is taken into account.

\section{References}

Bahcall J., Tremaine S., 1981, ApJ, 244, 805.

Bahcall N. A., Lubin L. M., Dorman V., 1995, ApJ, 447, L81.

de Vaucouleurs G., 1975, in Galaxies and the Universe, eds. Sandage A. et al., Univ. Chicago Press, p. 557.

Garcia A. M., Paturel G., Bottinelli, L., 1993, ApJS, 389, 68.

Geller M. J., Huchra J.P., 1983, ApJS, 52, 61.

Karachentsev I. D., 1994, Astron. Astrophys. Trans. 6, 1.

Karachentsev I. D. Binary Galaxies. Nauka, Moscow, 1987 (in Russian).

Karachentseva V. E., Karachentsev I. D., Lebedev V. S. 1988, Izvestija SAO, 26,42 .

Karachentsev I. D., Karachentseva V. E., Lebedev V. S. 1989, Izvestija SAO, $27,67$.

Karachentsev I. D., Shcherbanovskii A. L., 1978, Soobschenija SAO, 24, 5.

Karachentseva V. E., Karachentsev I. D., Shcherbanivskii A. L. 1979, Izvestija $\mathrm{SAO}, 11,3$.

Magtesian A. 1988, Astrofizika 28, 150.

Nolthenius R. 1993, ApJS85, 1.

Makarov D. I., Karachentsev I.D., 2000, Catalog of Small Galaxy Groups in the Local Supercluster (in preparation).

Tully R. B., 1987, ApJ, 321, 280.

Tully R. B., 1988, Nearby Galaxy Catalogue, Cambridge Univ. Press.

Vennik J., 1986, Dissertation, Tartu.

Zwicky F., Herzog E., Wild P., Karpowicz M., Kowal C., 1968, Catalog of Galaxies and Clusters of Galaxies: VV. I-VI, Cal. Inst. Tech., Pasadena. 